\title{
Análisis preliminar de un servicio expreso derivado de la ruta P8 del Sistema Integrado de Transporte Masivo (SITM) Metrolínea en Bucaramanga
}

\author{
Sergio Alonso González Jiménez \\ Grupo GEOMÁTICA, Universidad \\ Industrial de Santander \\ cheo.gonzalez@hotmail.com
}

\author{
Carlos Javier Leandro \\ Hernández Moreno \\ Grupo GEOMÁTICA, Universidad \\ Industrial de Santander \\ cajalehemo@gmail.com
}

\author{
Daniel Eduardo Rueda Sosa \\ Grupo GEOMÁTICA, Universidad \\ Industrial de Santander \\ danied01993@hotmail.com
}

(Tipo de Artículo: Investigación Científica y Tecnologica. Recibido el 01/08/2016. Aprobado el 15/09/2016)

\begin{abstract}
Resumen. En este artículo se presenta un análisis preliminar para la implementación de un servicio expreso derivado de la ruta P8 del SITM Metrolínea en el área metropolitana de Bucaramanga Colombia. Se revisaron los antecedentes referentes a metodologías adoptadas en servicios expresos o servicios de parada limitada, identificando factores influyentes para la efectiva implementación del servicio. Los datos que respaldan el análisis se han tomado de encuestas realizadas a los usuarios de la ruta en estudio y de observaciones efectuadas en paradas y estaciones del SITM, vehículos de la ruta y el corredor de la ruta. Se analizan las posibles modalidades de servicio expreso junto con la evaluación de las condiciones actuales de la ruta P8, del SITM y del área metropolitana. Se concluye que el área metropolitana de Bucaramanga y el SITM necesitan mejoras, primordialmente en infraestructura, para la efectiva implementación de un servicio expreso que cumpla con la demanda y necesidades de los usuarios.

Palabras clave. Análisis preliminar, autobús de tránsito rápido, servicio de paradas limitadas, servicio expreso, transporte público.

\section{Preliminary analysis of an express route service derived from route P8 of the Bus Rapid Transit System (BRT) Metrolínea in Bucaramanga}

\begin{abstract}
This paper presents a preliminary analysis for the implementation of an express service derived from route $P 8$ of the BRT Metrolínea in the metropolitan area of Bucaramanga Colombia. Background concerning methodologies adopted in express services or limited stop services were reviewed, identifying influential factors for effective implementation of the service. The data supporting the analysis were taken from surveys of the users of the studied route and observations in the BRT stops and stations, vehicles and route corridor. Possible modalities for express service are analyzed along with the assessment of current conditions of route $P 8$, the $B R T$ and the metropolitan area. It is concluded that the metropolitan area of Bucaramanga and the BRT system need improvements, primarily in infrastructure, for the effective implementation of an express service that meets the demands and needs of users.
\end{abstract}

Keywords. Preliminary analysis, bus rapid transit (BRT), limited-stop services, express services, public transit.

\section{Introducción}

Los Sistemas Integrados de Transporte Masivo (SITM) han surgido como una respuesta de los gobiernos frente a los problemas del transporte público en los países, con el fin de mejorar la calidad de vida, impulsar el desarrollo urbano y la economía regional. En las principales ciudades de Colombia, se han implementado estas iniciativas; por ejemplo: TransMilenio (Bogotá), TransMetro (Barranquilla), Metroplús (Medellín), M.I.O. (Cali) y Metrolínea (Bucaramanga). Este último fue inaugurado, en su primera fase, en diciembre de 2009, siguiendo la política de estado de la implementación de proyectos SITM para ciudades de más de 600000 habitantes, bajo un esquema de participación público - privada [1].

Sin embargo, debido al crecimiento de la población, el cambio en las condiciones de las vías y otros factores, algunos SITM no pueden suplir la demanda de pasajeros, causando congestión en el sistema, demoras en los servicios e inconformidad por parte de los usuarios. Para solucionar parte de esta problemática, muchos SITM implementan los servicios expresos, que en general se pueden definir como servicios que atienden a un subconjunto de los paraderos de un determinado corredor; son una estrategia de operación que puede ser beneficiosa tanto para los operadores como para los usuarios. Una reducción en el número de paradas beneficia a los pasajeros, y se refleja en una disminución en los tiempos de viaje [2].
La referencia [2] muestra la creación de un servicio expreso con un problema altamente complejo debido al estudio especializado necesario, como el uso de modelos y algoritmos, para el diseño de la ruta. El fin último de la implementación, de este tipo de alternativa, es disminuir los tiempos de viaje, incrementar la capacidad de un corredor y mejorar la percepción que tiene el usuario, de tal manera, que el servicio y el sistema se vuelvan atractivos [3].

Ahora bien, la referencia [4] plantea algunos factores que contribuyen a la implementación efectiva de un servicio de paradas limitadas; que generalmente, implican elementos de tiempo y geografía, volumen de pasajeros, patrones de viajes de usuarios, coordinación con el servicio local, entre otros. No obstante, estudios referentes ej. $[3,5,6]$ modelan o diseñan un servicio de bus de paradas limitadas para un corredor, sin considerar el cumplimiento de criterios necesarios para que el servicio expreso sea exitoso y efectivo.

Este artículo presenta un análisis preliminar para una posible implementación de un servicio expreso, derivado de la ruta P8 del SITM Metrolínea. Este análisis se fundamenta en la determinación de las falencias que provocan que el servicio expreso no logre su máxima productividad. Por lo tanto, se busca reunir información que permita una evaluación de las necesidades de su sistema, y de este modo, garantizar que el servicio expreso adoptado cumpla algunos factores 
indispensables para aumentar la eficiencia y confiabilidad de su servicio.

El documento se organiza de la siguiente manera: en primer lugar, la sección de antecedentes consiste en revisar las diversas metodologías que se han desarrollado para implementar los servicios expresos en algunas ciudades del mundo y la identificación de criterios importantes para el éxito de la implementación de un servicio expreso; segundo, se muestra la metodología adoptada para llevar a cabo el estudio; posteriormente, se procesa la información obtenida a partir de las encuestas, con el fin de caracterizar la muestra y exponer la postura de los encuestados frente a la posibilidad de un servicio expreso; para continuar, se analiza la evaluación de las condiciones actuales de la ruta $\mathrm{P} 8$, basados en estudios de campo realizados previamente; por último, se exponen las principales conclusiones y demás extensiones para el análisis realizado.

\section{Antecedentes}

\subsection{Revisión bibliográfica}

El diseño de sistemas de transporte adecuados, teniendo en cuenta la estructura de cada ciudad, es un tema clave en el desarrollo urbano actual; una mala planificación del diseño implica resultados negativos, empeorando progresivamente la calidad de los sistemas. Además, las diferentes implementaciones del transporte público favorecen o contrarrestan la calidad de vida de una ciudad [7].

Como solución al problema de redes de tránsito (Transit network problem, TNP) se han establecido lineamientos generales en el proceso de planteamiento del tránsito, dividiéndolo en cinco pasos: el primero, corresponde al diseño de cada ruta nueva y análisis de las existentes; segundo, la determinación de las frecuencias de las rutas; posteriormente, la determinación de los puntos de transferencia y cronograma de las rutas; para continuar, la asignación de la flota de vehículos; y finalmente, la asignación de personal y demás recursos para cada línea [8].

Por otra parte, nuevos desarrollos en sistemas de transporte involucran la interacción entre controladores de software, redes de comunicación y dispositivos físicos, que hacen indispensable el uso de tecnologías automatizadas, como herramientas de diseño de sistemas de control asistidas por computador, que verifiquen y validen los diseños adoptados garantizando su seguridad y fiabilidad [9].

Un objetivo importante en la planificación del tránsito, es mejorar la velocidad y confiabilidad de servicios de buses urbanos [4]; además, para el mejoramiento del servicio, el mayor enfoque debe ser dirigido a los tres atributos controlados por el operador: tiempo de llegada del bus a la parada, combinado con la frecuencia y la desconfianza del servicio [10]. Después de lo anterior expuesto, existe una relación directa entre la cantidad de paradas y el tiempo de viaje; es por ello, que un servicio es más deseable por el usuario, si los tiempos de ingreso, egreso y permanencia en el vehículo son mínimos [10], [11]. Como consecuencia de la reducción del tiempo en marcha del bus, se espera un aumento en el número de usuarios de la ruta, y, por ende, la satisfacción de ellos [10], [12].

Disminuir la cantidad de paradas dentro del corredor de una ruta, es una de las estrategias más eficaces para que el tiempo de recorrido disminuya y que la velocidad de viaje aumente [3]. Por tal razón, los servicios expresos son vistos como una alternativa importante y atractiva para mejorar el desempeño de los sistemas de transporte público.

Entre las estrategias de operación de servicios expresos, se encuentran [13] y [4]: en [13], se proponen servicios cortos (short turn), deadheading y servicios expresos o servicios de paradas limitadas (express services, limited-stop service); [4] muestra que los servicios expreso también se pueden realizar teniendo en cuenta alguno de los siguientes modos de operación: el primero, consiste en que las paradas del servicio expreso deben estar ubicadas en intervalos de ocho a diez cuadras, equivalentes a 0.5 millas ( 800 metros aprox.); otro, es que las paradas estén ubicadas cerca de instituciones o lugares representativos (centros educativos, centros comerciales, etc.); y finalmente, consiste en que el servicio expreso se detenga únicamente en las paradas con un alto número de entrada y salida de usuarios; entre tanto, ej. [5], [14]-[16] proponen modelos heurísticos para encontrar soluciones desde la perspectiva de la programación matemática, producto de la combinación de diferentes variables. 


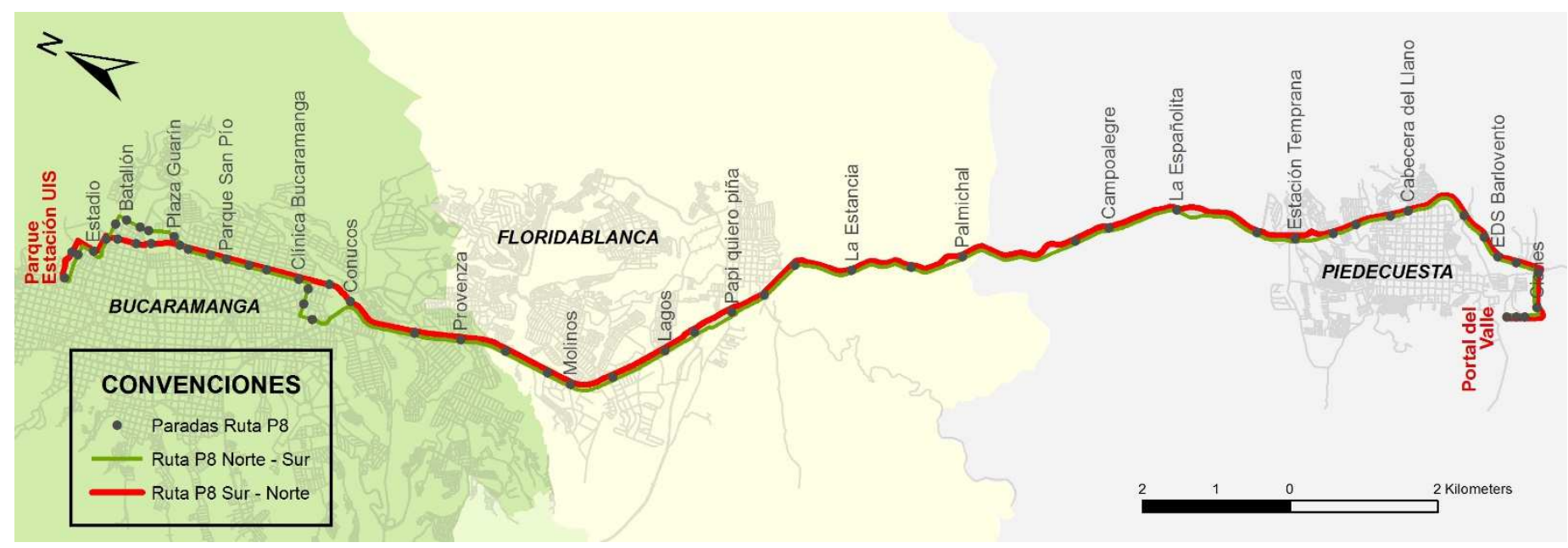

Figura 1. Ruta P8 SITM Metrolínea. Fuente: Adaptado de [17].

Tabla 1. Factores que afectan los tiempos de viaje de tránsito Fuente: adaptado de [3]

\begin{tabular}{|c|c|}
\hline Variables & Descripción \\
\hline Distancia & Longitud de segmento. \\
\hline Intersecciones & Número de intersecciones con semáforo. \\
\hline Paradas de bus & Número de paradas de bus. \\
\hline Abordaje & Número de pasajeros abordando. \\
\hline Desmontaje & Número de pasajeros desbordando. \\
\hline Tiempo & Periodo de tiempo. \\
\hline Conductor & Experiencia de conductor. \\
\hline $\begin{array}{l}\text { Periodo } \\
\text { servicio }\end{array}$ & $\begin{array}{l}\text { Cuánto tiempo de servicio lleva el conductor } \\
\text { en el periodo de estudio. }\end{array}$ \\
\hline $\begin{array}{l}\text { Demora } \\
\text { despacho }\end{array}$ & $\begin{array}{l}\text { Tiempo de despacho observado menos el } \\
\text { tiempo programado. }\end{array}$ \\
\hline $\begin{array}{l}\text { Tiempo } \\
\text { demora } \\
\text { parada }\end{array}$ & $\begin{array}{l}\text { Tiempo perdido en las paradas basado en la } \\
\text { configuración del bus (pisos bajos, etc.) }\end{array}$ \\
\hline $\begin{array}{l}\text { Eventos } \\
\text { recurrentes }\end{array}$ & Uso de rampa, apertura de puente, etc. \\
\hline Dirección & Servicio de entrada o salida. \\
\hline Clima & Condiciones relacionadas con el clima. \\
\hline Carretera & Características de viaje. \\
\hline $\begin{array}{l}\text { Ambiente } \\
\text { operación }\end{array}$ & Congestión. \\
\hline
\end{tabular}

Por otro lado, las condiciones de operación no han sido el enfoque que se le ha dado a los estudios recientes de servicios expresos. No obstante, [4] fue uno de los primeros en identificar factores clave para el funcionamiento eficiente de un servicio expreso, generalmente involucrando elementos de tiempo y geografía, como: los servicios expresos operan más efectivamente cuando su recorrido se realiza en vías más anchas, con un nivel de servicio $\mathrm{C}$ o mejor, cuando operan en vías donde existe semaforización, cuando operan con una distancia entre paradas de 0.5 millas, y cuando el servicio no opera paralelo a una ruta de metro o tren cercana. Por su parte, [3] plantea un resumen de factores que afectan los tiempos de viaje, reflejados en la Tabla 1, pero, algunos de ellos no son tenidos en cuenta al momento del diseño de la ruta.
En consecuencia, vale la pena una evaluación de factores que han influenciado el éxito de rutas expreso en algunas ciudades del mundo, y a su vez, la consideración de algunos problemas que aún se siguen presentando a medida que continúan evolucionando los servicios expreso [4]. Al mismo tiempo, debido a que el servicio de buses está vinculado directamente al comportamiento de los usuarios, es fundamental evaluar la percepción y la satisfacción de ellos, luego de la implementación del servicio [3].

\subsection{Generalidades del servicio ruta P8 del SITM Metrolínea}

La ruta P8 del SITM Metrolínea hace parte del conjunto de trayectos que ofrece este servicio público, abarcando parte de las cabeceras municipales de Piedecuesta, Floridablanca y Bucaramanga.

Su recorrido inicia en Portal del Valle, municipio de Piedecuesta, en sentido sur - norte, cubriendo la demanda usuarios de Piedecuesta, parte de Floridablanca y del sector oriental de Bucaramanga, conocido como "Cabecera", hasta Parque Estación UIS y retomando por un trayecto paralelo en sentido norte sur hasta su lugar de origen, como se evidencia en la Figura 1.

En base a información suministrada por el ente gestor del SITM, se conoce que el trayecto de la ruta P8 "Cisnes - Carrera 33 - UIS", tiene una longitud de 49.8 kilómetros y ciclos de recorrido de 2:20 horas aproximadamente, cubriendo 99 puntos de parada distribuidos a lo largo del corredor; además, cuenta con 12 vehículos padrones con capacidad nominal de 90 y real de 100 pasajeros, donde, diariamente se realizan 10.500 validaciones en el sistema de torniquetes (conteo automático de pasajeros, APC) implementado dentro de los buses, pero sin tener en cuenta las validaciones de los usuarios que ingresan al vehículo por medio de las estaciones del SITM. 


\section{Metodología}

La metodología se resume en cinco etapas, las cuales componen la estructura general (Figura 2) que permite realizar los procedimientos de una manera organizada y eficiente.

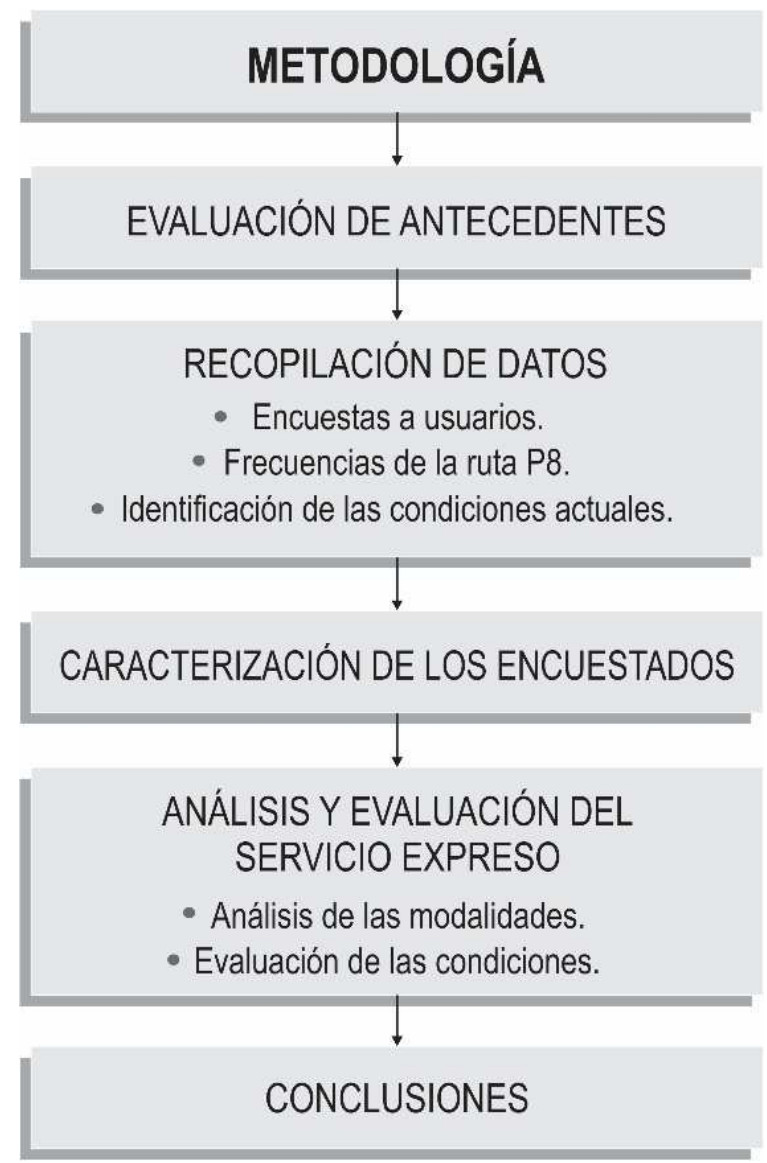

Figura 2. Estructura general de la metodología. Fuente: Autores.

\subsection{Evaluación de antecedentes}

El producto de la evaluación de antecedentes es apreciable en la sección 2 de este documento, y enmarca la revisión de literatura y las generalidades de la ruta $\mathrm{P} 8$ del SITM Metrolínea.

Como primera medida, se reúnen documentos de investigación aplicados a los servicios expresos y a sistemas de transporte público, para evaluar las metodologías adoptadas en cada estudio, y a su vez, identificar variables que influyen en el diseño eficiente de un servicio expreso. Por otro lado, se examinan las generalidades de la ruta en estudio, identificando el corredor principal, paradas asociadas, oferta de vehículos, demanda de usuarios, entre otros.

\subsection{Recopilación de datos}

En lo que se refiere a la recopilación de datos, el eje central se enmarca en la información obtenida de los usuarios de la ruta P8, el esquema operacional de la ruta y la identificación de las condiciones actuales del corredor y la ruta.
Para obtener la información primaria de los usuarios de la ruta P8, se estructura una encuesta que registra: la razón de uso de la ruta, los viajes realizados, estaciones o paradas de entrada y salida, la opinión personal frente a la posible implementación de un servicio expreso, entre otros. Cabe aclarar que, para la formulación de las preguntas, los puntos de parada utilizados son los más conocidos por los usuarios; en algunos casos se ha determinado mencionar centros de interés cercanos al punto de parada. Las encuestas se realizan en dos modalidades: la primera, de manera virtual utilizando la herramienta de Google Forms; y la segunda, aplicadas dentro de la ruta y las principales estaciones del SITM. Por último, las encuestas obtenidas se procesan, para descartar las no confiables, obteniendo 343 respuestas válidas de usuarios.

Por otro lado, el esquema operacional de la ruta está enfocado inicialmente en las frecuencias de los buses que conforman el servicio P8 del SITM Metrolínea; para ello, se registra el tiempo entre vehículos del sistema para un día típico y atípico en tres estaciones principales dentro del corredor: Parque Estación UIS (extremo de la ruta), Provenza y Estación Temprana, para luego compararlas con los despachos realizados en Portal del Valle (inicio y fin de la ruta).

Finalmente, teniendo en cuenta las variables asociadas en la revisión bibliográfica, se identifican las condiciones actuales del corredor y la ruta P8, como: infraestructura vial y del sistema, tiempos de abordaje, características del corredor, ambiente de operación, comportamiento de los usuarios, entre otros; para su posterior evaluación.

\subsection{Caracterización de los encuestados}

El resultado de esta etapa se expone en la sección 4 de este documento como producto de la tabulación y el procesamiento de la información obtenida de las encuestas realizadas. Se representan los datos obtenidos mediante gráficas, facilitando la interpretación para su posterior análisis.

\subsection{Análisis y evaluación del servicio expreso}

Con referencia a la evaluación de antecedentes y a la recopilación de datos, esta sección comprende el análisis de las diferentes modalidades de servicio expreso que pueden darse en el caso estudio y la valoración de las condiciones actuales del corredor y la ruta P8.

El análisis de las modalidades se basa en teorías de servicios expresos planteada por [13], y los criterios adoptados están soportados en la demanda de usuarios obtenida en las respuestas de las encuestas. Adicionalmente, a criterio de los autores se propone una modalidad de servicio expreso que suple la demanda identificada en las respuestas de los usuarios de la ruta.

Por otro lado, la evaluación de las condiciones actuales del corredor y la ruta P8 se realiza bajo los criterios mencionados en la revisión bibliográfica. Esta valoración cualitativa se enfoca en las falencias que se 
presentan actualmente y en las mejoras necesarias para una eficiente implementación de un servicio expreso derivado de la ruta P8.

\section{Caracterización de los encuestados}

Con el fin de analizar el posible comportamiento de los usuarios de la ruta P8 ante la implementación de un servicio expreso, es importante identificar características generales de la muestra obtenida. Los resultados de la encuesta se muestran a continuación, luego de su tabulación y procesamiento.

\subsection{Información preliminar}

Como se espera, los encuestados están constituidos por 174 mujeres y 169 hombres, es decir, cada género corresponde aproximadamente al $50 \%$ de la muestra, los cuales están distribuidos en rangos de edad como muestra la Figura 3, destacando que tan sólo un $10 \%$ de los encuestados son mayores de 30 años.

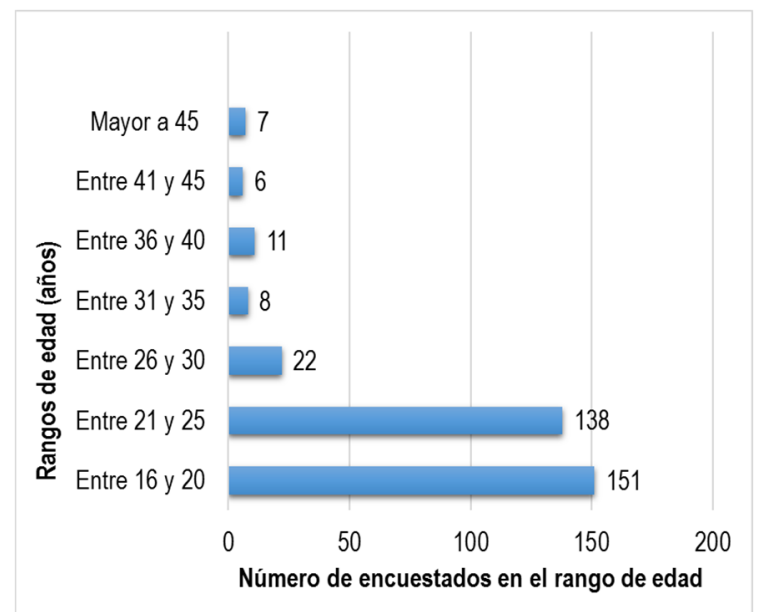

Figura 3. Representación gráfica del rango de edad de los encuestados. Fuente: Autores.

En lo referente a la ocupación de los usuarios seleccionados al azar, la Figura 4 agrupa las diferentes actividades realizadas por los encuestados. Dentro de la muestra, el número elevado de estudiantes es debido inicialmente a la presencia de universidades y colegios paralelos al corredor, tales como: Universidad Industrial de Santander, Colegio Tecnológico "Dámaso Zapata", Universidad Cooperativa de Colombia, Universidad Santo Tomás (Floridablanca) y la Universidad Pontificia Bolivariana (Piedecuesta), entre otros.

\subsection{Uso de la ruta P8}

Para esta sección se solicitó la frecuencia con la que cada usuario usa la ruta P8 (Figura 5), y de esta manera determinar el horario que pueda tener el servicio expreso, dicho de otra manera, la pregunta fue planteada para estudiar la viabilidad de la operación de una ruta expreso los fines de semana.

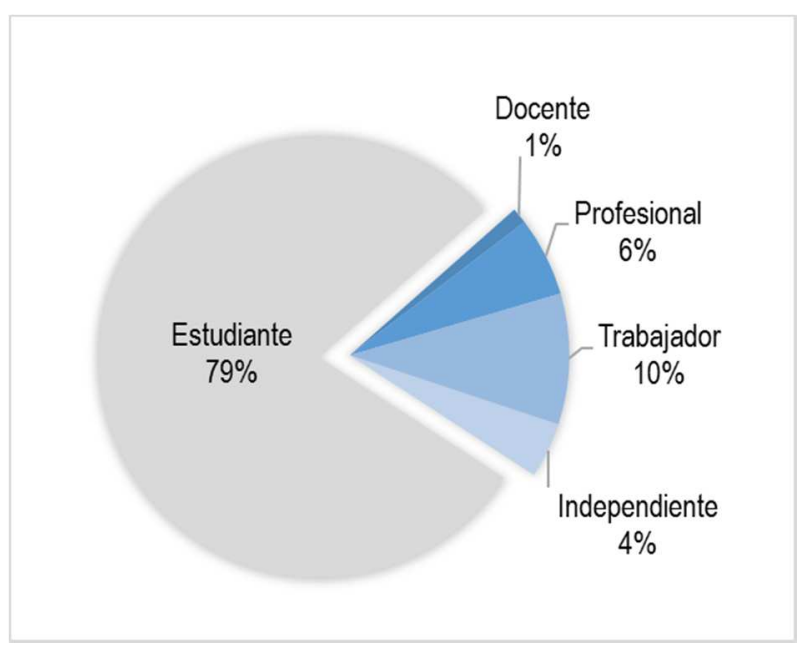

Figura 4. Representación porcentual de la ocupación de los encuestados. Fuente: Autores.

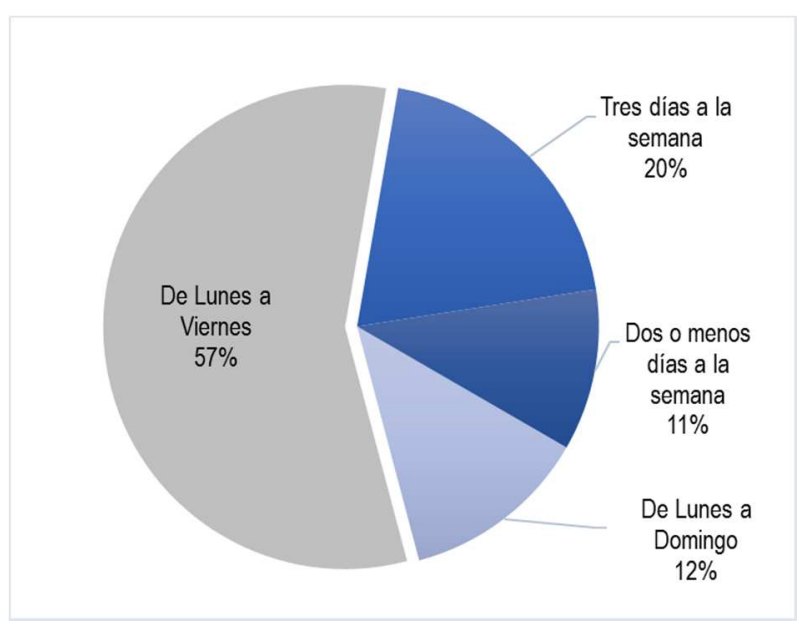

Figura 5. Representación porcentual del uso semanal de la ruta P8 de los encuestados. Fuente: Autores.

\subsection{Razón de uso}

La Figura 6 muestra la razón por la cual los usuarios encuestados toman la ruta P8. Se evidencia que la ocupación va ligada con la razón de uso de la ruta, en donde un $72 \%$ de los encuestados realizan su viaje con fines académicos. Se infiere que, en la posible existencia del servicio expreso, se puede tener la opción de suspender temporalmente el servicio en temporadas de receso académico.

Además, se solicitó cuántas veces al día utilizan el servicio de la ruta P8 (Figura 7), las paradas y el horario en el que ingresan y egresan del vehículo. Esto, con el fin de conocer el flujo de pasajeros dentro del sistema con enfoque en la ruta de estudio y de esta manera determinar las estaciones con mayor demanda y conocer horarios picos del servicio. 


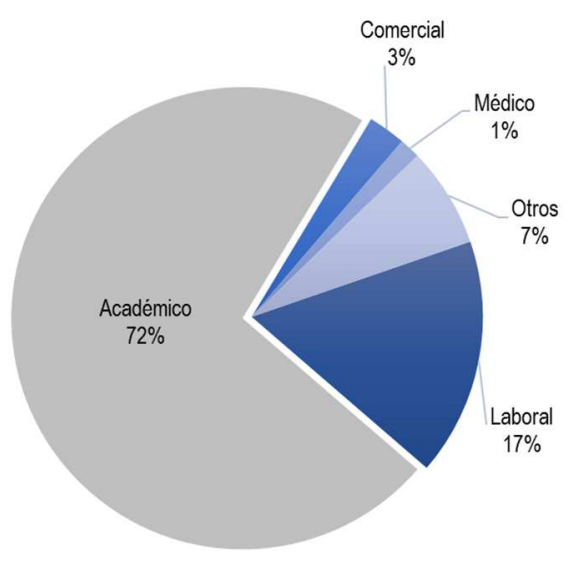

Figura 6. Representación porcentual del motivo por el cual los encuestados usan la ruta en estudio. Fuente: Autores.

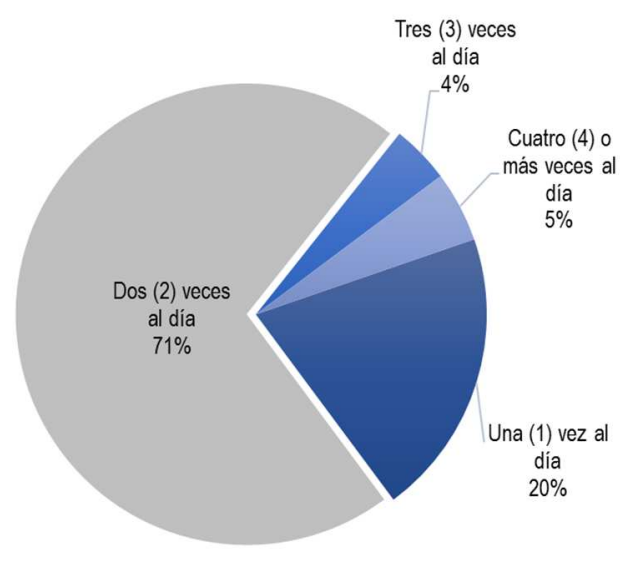

Figura 7. Representación porcentual de la frecuencia diaria del uso de la ruta P8. Fuente: Autores.

\subsection{Viabilidad}

El objetivo de esta sección es entender el comportamiento de usuarios en caso de una posible implementación de un servicio expreso. Se proponen dos casos (necesidad de hacer transbordos para acceder a la ruta y menor frecuencia en comparación con las rutas existentes), los cuales se muestran en las Figuras 8 y 9 respectivamente y que posteriormente serán respaldo en la sección de evaluación de las condiciones actuales de la ruta en estudio.

Por otro lado, se indaga si consideran que las estaciones de puerta derecha recientemente colocadas en la Carrera 33 han contribuido con las condiciones de ingreso al SITM y los resultados plasmados en la Figura 10 muestran una recepción negativa.

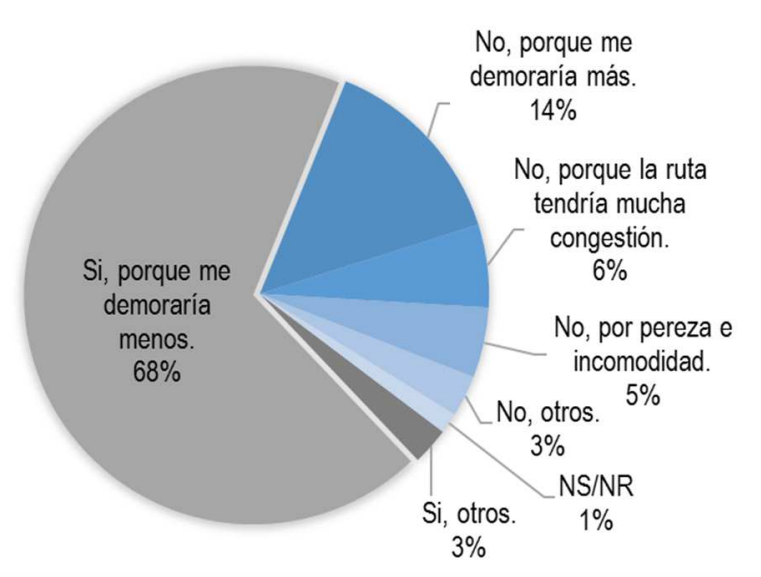

Figura 8. ¿Si se propone una ruta que haga más rápido el recorrido del P8 y que no haga todas las paradas, estaría dispuesto a tomarla, así esto implique hacer un trasbordo? Fuente: Autores.

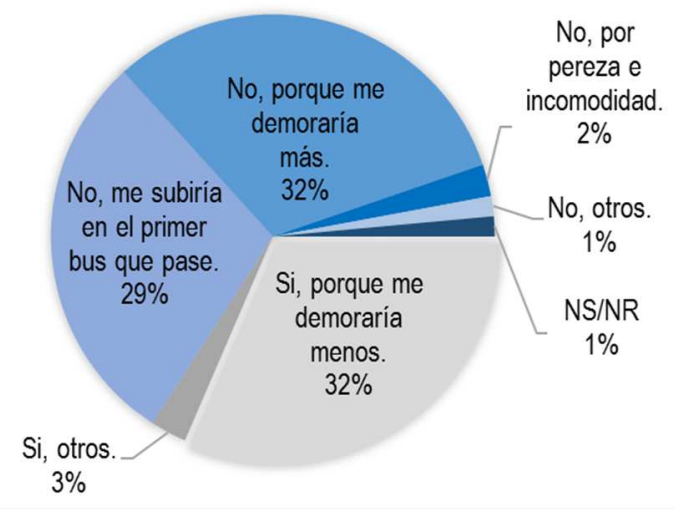

Figura 9. ¿Estaría dispuesto a esperar la ruta que hace más rápido el recorrido del $\mathrm{P8}$, así la ruta no pase tan seguida como el P8? Fuente: Autores.

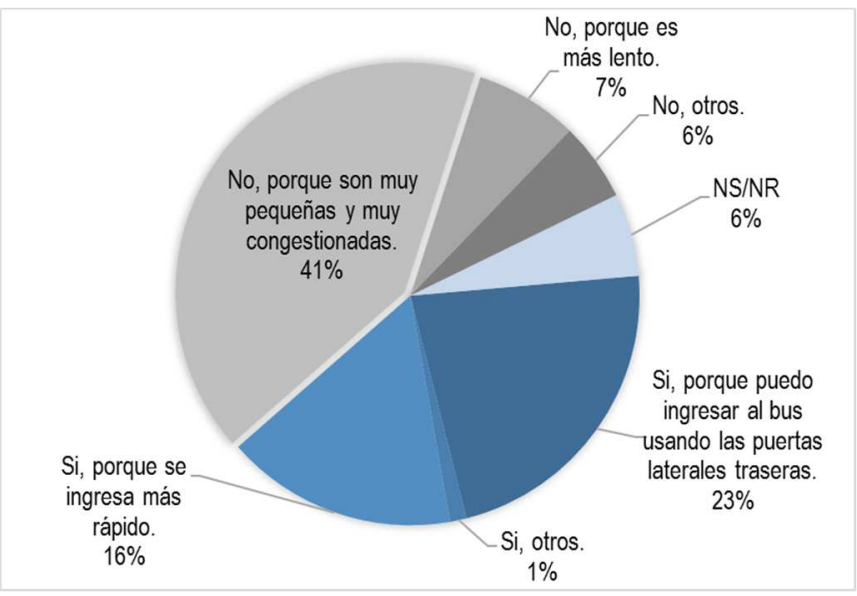

Figura 10. ¿Cree usted que los vagones colocados en algunas paradas de la Carrera 33 han mejorado las condiciones para ingresar al SITM Metrolínea? Fuente: 


\section{Análisis de diseño del servicio expreso}

El análisis se lleva a cabo en dos etapas: primero, se analizan las modalidades de servicio expreso aplicadas a la ruta en estudio; y segundo, se evalúan las condiciones actuales de la ruta en base a los factores encontrados en la revisión de literatura.

\subsection{Modalidades de servicio expreso aplicados a la ruta en estudio}

Se presentan las diferentes modalidades de servicio expreso identificadas en la revisión bibliográfica, y a su vez, se describe el modo operacional de cada una. Sumado a esto, en cada modalidad se propone un esquema de funcionamiento, adaptado a la ruta en estudio.

\subsubsection{Servicios cortos (short turn)}

Esta modalidad consiste en que una porción de vehículos realice viajes más cortos dentro del corredor, es decir, que no operen a lo largo de la longitud total de la ruta base. Con referencia a lo anterior y teniendo en cuenta la investigación en campo realizada, se evidencia que ya existe esta modalidad con dos rutas del SITM que operan dentro del corredor de la ruta P8: las rutas troncales P7 y P13, tal como se plantea en la Figura 11.

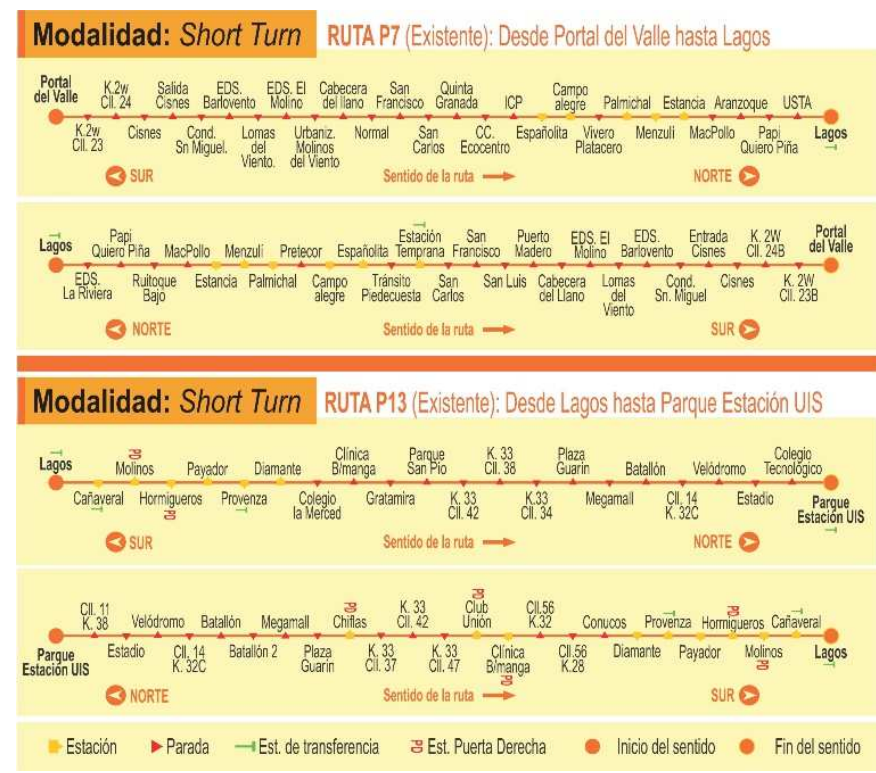

Figura 11. Esquema existente de la modalidad short turn de la ruta P8. Fuente: Autores.

Para el correcto funcionamiento de esta modalidad, es necesaria la creación o adecuación de un retorno en cada extremo de los corredores de las rutas que sirven bajo esta modalidad, con el fin de que los vehículos del servicio propuesto puedan cambiar de sentido sin depender del flujo vehicular o condiciones de movilidad ajenas al SITM, e iniciar lo antes posible su recorrido en el nuevo sentido.

\subsubsection{Deadheading}

Este modo de servicio expreso está enfocado en corredores que presentan un desbalance en la demanda de usuarios entre ambos sentidos de operación, y consiste en que una porción de los vehículos se desplaza en el sentido menos cargado sin recolectar pasajeros en las paradas intermedias del corredor, con el fin de comenzar lo antes posible un nuevo viaje en el sentido más cargado.

Ante la situación planteada, se toma como base el análisis de las trayectorias de usuarios obtenidas en las encuestas. Inicialmente, se observa que las paradas intermedias presentan un número considerable de usuarios, y a primera instancia se propone que no es viable omitir estas paradas, debido a que, sin importar la hora del día, las paradas intermedias presentan una actividad de usuarios como lo muestra la Figura 12.

Por otro lado, se tiene en cuenta la configuración del corredor, donde los usuarios se desplazan en la mañana desde el sector sur (zonas de alta densidad de viviendas) hacia el sector norte que presenta universidades, clínicas, centros de comercio y otros sitios de actividad adyacentes al corredor. Con base a lo anterior, se propone una ruta con modalidad deadheading que en las mañanas realice todas las paradas en sentido sur - norte y regrese a su punto de inicio sin realizar las paradas intermedias, con el fin de iniciar un nuevo recorrido lo antes posible; y en la tarde, se invierta el comportamiento realizando todas las paradas en sentido norte - sur como se evidencia en la Figura 13.

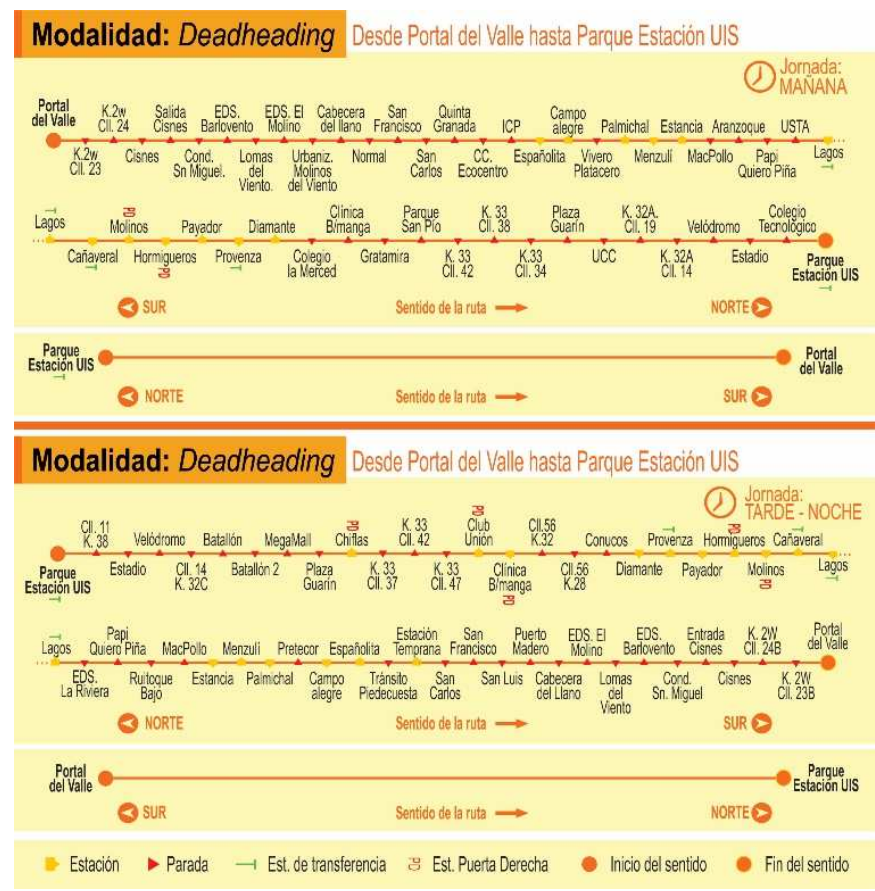

Figura 13. Esquema operacional propuesto para la modalidad deadheading. Fuente: Autores. 
Ingenierías USBMed, Volumen 7, No. 2, Julio-Diciembre 2016

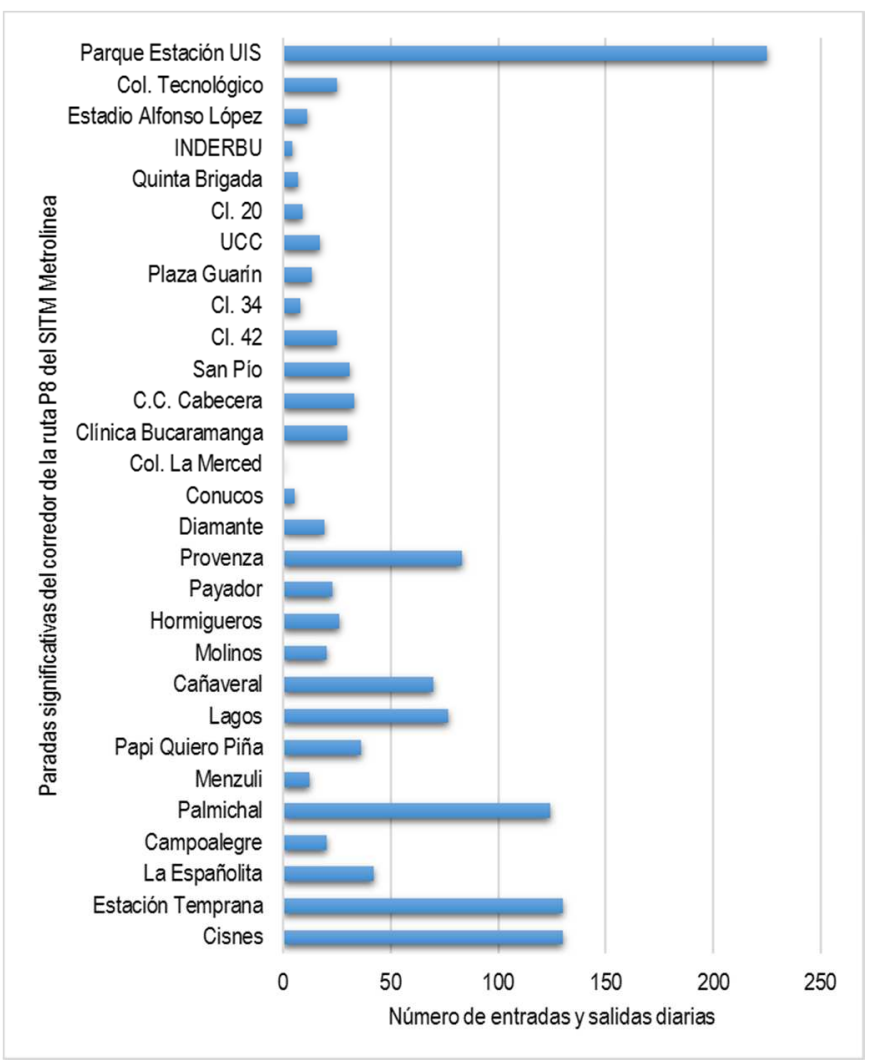

Figura 12. Relación entre paradas significativas y flujo de usuarios de la ruta P8. Fuente: Autores.

Esta modalidad exige que, para un óptimo funcionamiento del servicio se debe buscar una vía, sin importar si pertenece o no al corredor principal de la ruta en estudio, que permita el regreso de los vehículos que sirven el servicio expreso, lo más rápido posible.

\subsubsection{Servicios expresos o servicios de parada limitada (express services, limited-stop services)}

Consiste en que el vehículo se detenga únicamente en un subconjunto de las estaciones o paraderos que pertenecen a un corredor. Generalmente, esta modalidad se basa en las paradas con alta demanda y en las paradas más importantes según la percepción del usuario. Asimismo, este modo de operación plantea los servicios zonales y los servicios que atienden sólo dos paraderos: el primero, consiste en zonificar el corredor y atender exclusivamente aquellas que presenten alta demanda; el segundo, consiste en brindar un viaje rápido entre dos nodos de alta demanda.

\section{Servicio de paradas limitadas basado en las paradas con alta demanda:}

Producto de las encuestas a usuarios, la Figura 12 muestra las paradas con mayor actividad diaria de usuarios dentro del corredor de la ruta P8 del SITM. En base a lo anterior, el esquema operacional de esta modalidad se presenta en la Figura 14.

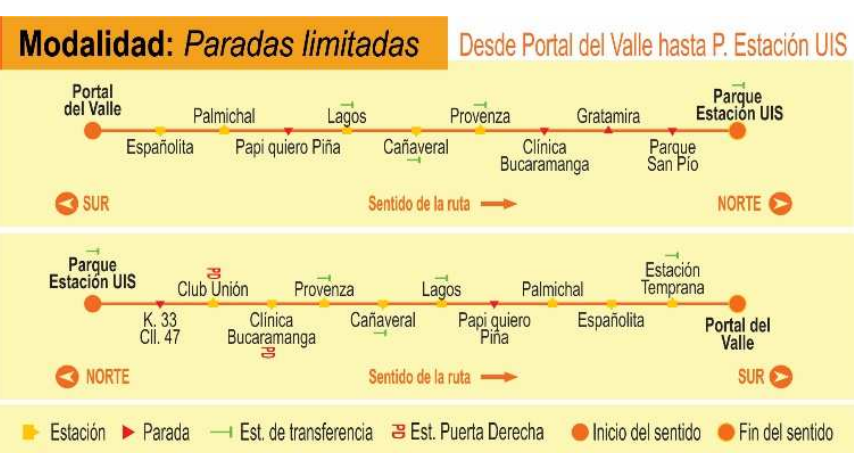

Figura 14. Esquema operacional propuesto para la modalidad paradas limitadas basada en puntos de parada con alta demanda. Fuente: Autores.

\section{Servicio de paradas limitadas basado en la percepción del usuario:}

Como parte de la encuesta realizada, en la Figura 15 se presenta la percepción de los usuarios referente a las paradas más importantes del corredor de la ruta P8 del SITM Metrolínea; y a partir de este criterio, se proponen las paradas que enmarcan esta modalidad (Figura 16).

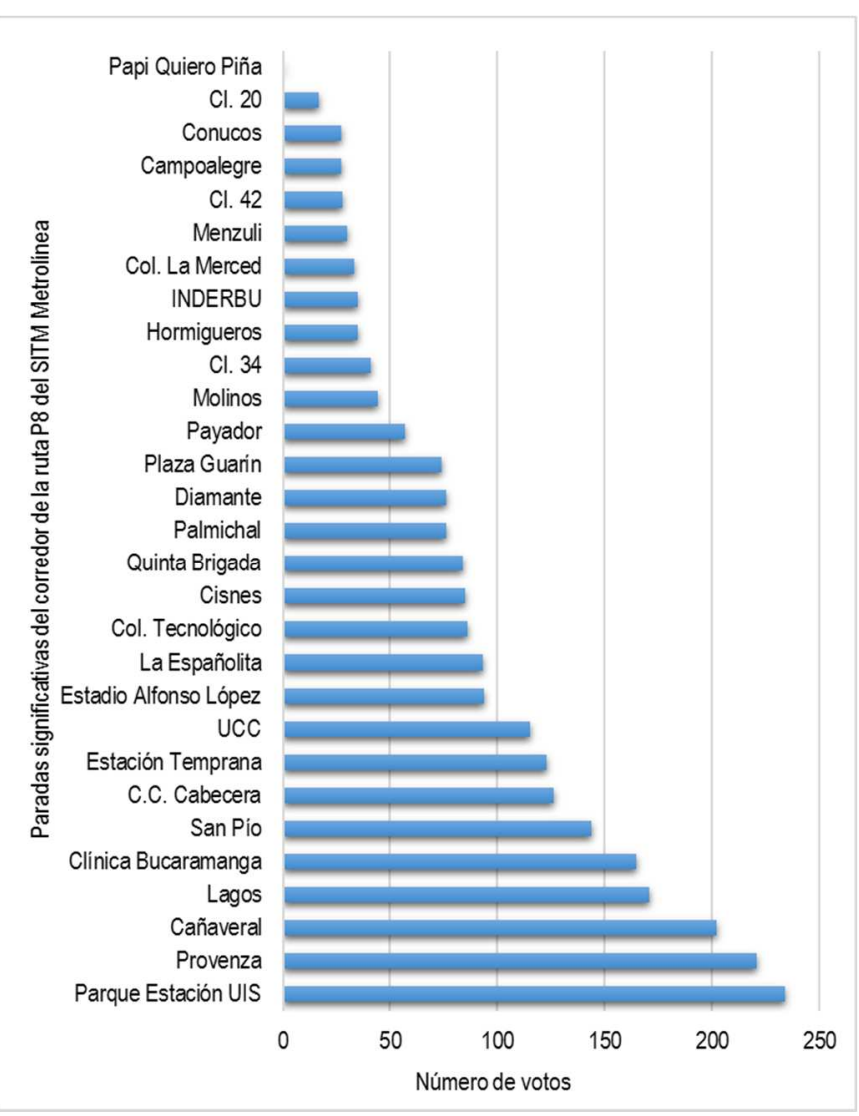

Figura 15. Percepción de los usuarios de las paradas más importantes de la ruta P8. Fuente: Autores. 


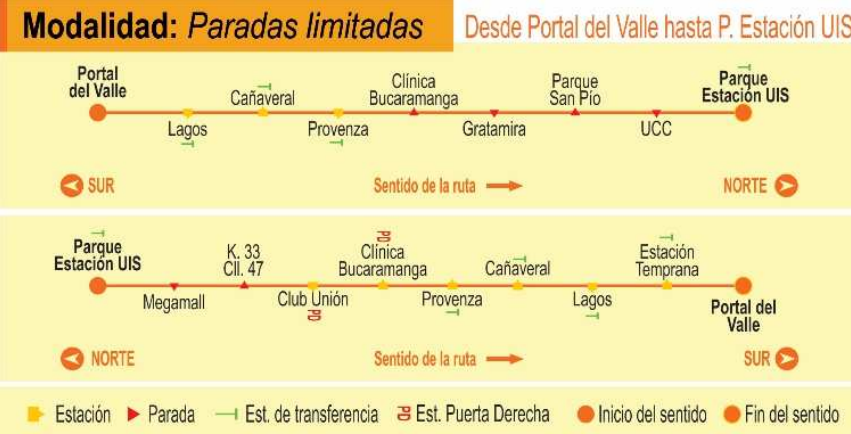

Figura 16. Esquema operacional propuesto para la modalidad paradas limitadas basada en percepción del usuario. Fuente: Autores.

\section{Servicios zonales:}

El primer paso para llevar a cabo esta metodología consiste en dividir en zonas el corredor de la ruta P8, teniendo en cuenta la demanda de usuarios observada en la recopilación de datos, e identificar las rutas troncales y pretroncales del SITM Metrolínea que presentan influencia, en por lo menos una parada ubicada dentro de cada zona, como lo indica la Tabla 2.

Tabla 2. Distribución de zonas dentro del corredor de la ruta P8. Fuente: Autores

\begin{tabular}{ll}
\hline Zonas & Descripción \\
\hline 1 & $\begin{array}{l}\text { Desde Portal del Valle hasta la parada C.C. } \\
\text { Ecocentro o su parada homóloga Estación } \\
\text { Temprana (Piedecuesta). }\end{array}$ \\
\hline 2 & $\begin{array}{l}\text { Desde la parada ICP o su homóloga Tránsito } \\
\text { Piedecuesta hasta la parada Ruitoque Bajo } \\
\text { o su homóloga Aranzoque. }\end{array}$ \\
\hline 3 & $\begin{array}{l}\text { Desde la parada Papi Quiero Piña hasta la } \\
\text { estación Diamante. }\end{array}$ \\
\hline 4 & $\begin{array}{l}\text { Desde la parada Colegio La Merced o su } \\
\text { homóloga Conucos hasta la parada UCC o } \\
\text { su homóloga Megamall. }\end{array}$ \\
\hline 5 & $\begin{array}{l}\text { Desde la parada Carrera 32A con Calle 19 o } \\
\text { su homóloga Batallón 2 hasta Parque } \\
\text { Estación UIS. }\end{array}$ \\
\hline
\end{tabular}

Para el servicio zonal se proponen las Zonas 1 y 4 como las más representativas del corredor ya que encierran los principales sectores de la ruta P8 (cabecera municipal de Piedecuesta y la Carrera 33 de Bucaramanga) en los que, además, se presenta menor cobertura de rutas del SITM. Finalmente, se propone que el servicio expreso bajo zonas, opere mediante el esquema que se presenta en la Figura 17.

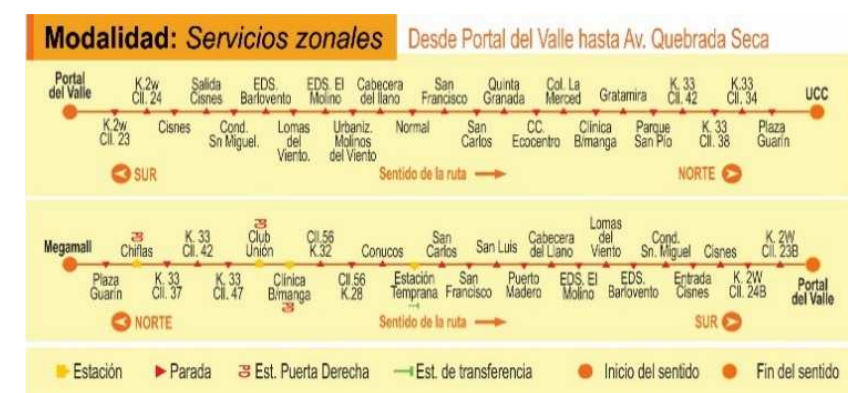

Figura 17. Esquema operacional propuesto para la modalidad servicio expreso basada en zonas. Fuente: Autores.

\section{Servicio de dos puntos:}

En lo que se refiere a este servicio, se continúa teniendo en cuenta la demanda de usuarios observada en la recopilación de datos, logrando identificar paradas con alta actividad diaria a lo largo del corredor, presentada en la Figura 12.

- Debido a que la solución se convierte en un problema combinatorio, se requiere un análisis más profundo para establecer las paradas definitivas de la posible ruta expreso. Sin embargo, se recomienda que los nodos definitivos estén dentro de los diferentes corredores de las rutas con modalidad short turn ya existentes (rutas P7 y P13). En este orden de ideas, se proponen los siguientes criterios para un análisis posterior: Uno de los nodos sea alguno o ambos extremos del corredor de la ruta P8 (Parque Estación UIS y/o Portal del Valle). Un ejemplo se plantea en la Figura 18(a).

- Uno de los nodos se encuentre ubicado en la cabecera municipal de Piedecuesta, y el otro dentro de una troncal principal de la ruta P8: la Carrera 33 de Bucaramanga. En la Figura 18(b) se muestra un ejemplo de este esquema.

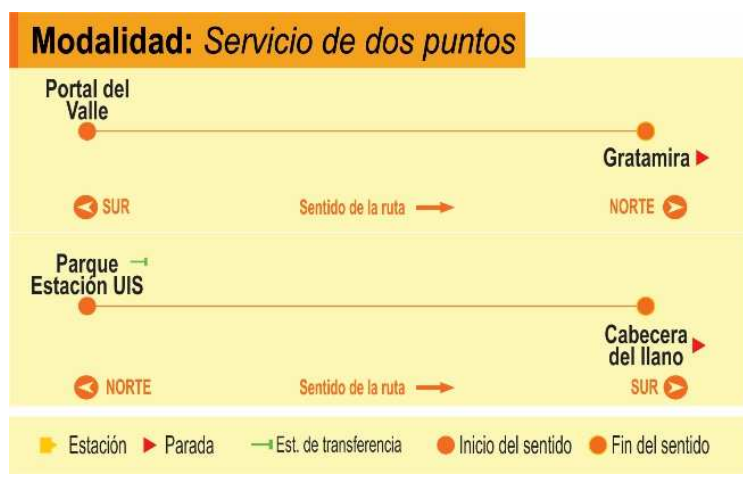

Figura 18 (a). 


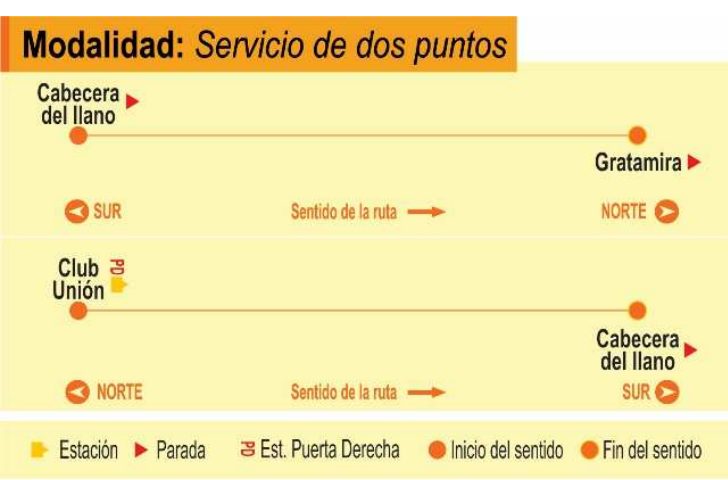

Figura 18 (b).

Figura 18. Esquemas operacionales propuestos para la modalidad servicio de dos puntos. Fuente: Autores.

\section{Propuesta de los autores:}

Tomando como referencia la recopilación de datos y la revisión bibliográfica se establece un servicio expreso que contempla algunos de los criterios expuestos anteriormente en las modalidades short turn, deadheading y servicios expresos zonales; además, se toman como referencia algunos puntos de parada cercanos a centros de salud, educativos y de comercio presentes en el área metropolitana. El fin último de esta propuesta es sintetizar las soluciones que cada modalidad brinda a los usuarios, representados en un solo servicio. Se propone el esquema de operación plasmado en la Figura 19.
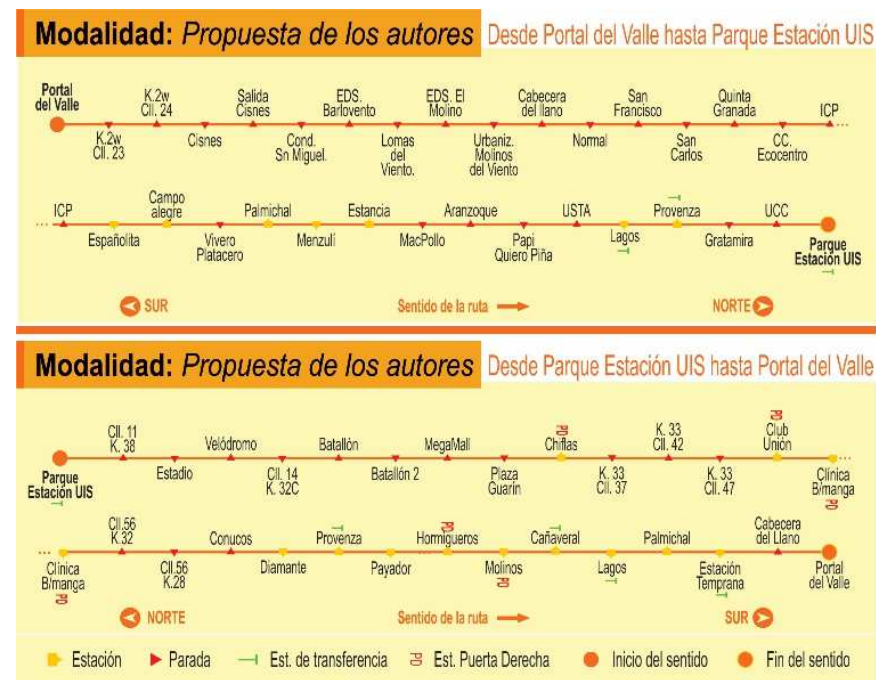

Figura 19. Esquema operacional propuesto por los autores. Fuente: Autores.

\subsection{Evaluación de las condiciones actuales de la ruta P8 del SITM Metrolínea}

De la evaluación de las condiciones actuales de la ruta P8 se resaltan factores que afectan la efectiva implementación de un posible servicio expreso derivado de la ruta en estudio, los cuales, se asocian a diferentes áreas de enfoque establecidas bajo criterio de los autores.

\subsubsection{Características del corredor}

Un primer paso para evaluar la efectividad de un posible servicio expreso es identificar algunas características del corredor, diferenciadas en: número y tipo de carriles e intersecciones.

\section{Número y tipo de carriles:}

La ruta P8 del SITM realiza su recorrido por algunas arterias viales del área metropolitana de Bucaramanga con una longitud total de 49.8 kilómetros. El $49 \%$ del corredor en estudio es un tramo segregado (exclusivo para el SITM Metrolínea) de un carril, y el $51 \%$ es un tramo denominado "mixto" (compartido con vehículos particulares, de transporte público colectivo convencional, motos, etc.) con predominancia de dos carriles por sentido a lo largo del corredor.

Si bien es cierto que, mientras la ruta hace su recorrido por el tramo mixto, las condiciones de movilidad están sujetas a factores que no pueden ser controlados por el sistema, afectando el tránsito y, por ende, la operación de la ruta.

\section{Número y tipo de intersecciones:}

El trayecto de la ruta en estudio contiene intersecciones sencillas (el corredor atraviesa una vía de un sólo sentido), intersecciones dobles (el corredor atraviesa una vía de dos sentidos) y algunas glorietas. Sin embargo, la influencia en la movilidad sólo se observa cuando la intersección no cuenta con semáforos reguladores de circulación, ya que, el vehículo debe llegar a un pare indefinido hasta que se le permita cruzar.

El corredor vial cuenta con seis intersecciones sencillas sin presencia de semáforo, tres intersecciones dobles sin semáforo y seis intersecciones de otras denominaciones (glorietas y accesos a autopistas) que afectan el tiempo de viaje del servicio. La Figura 20 evidencia una intersección doble sin semáforo presente en el recorrido.

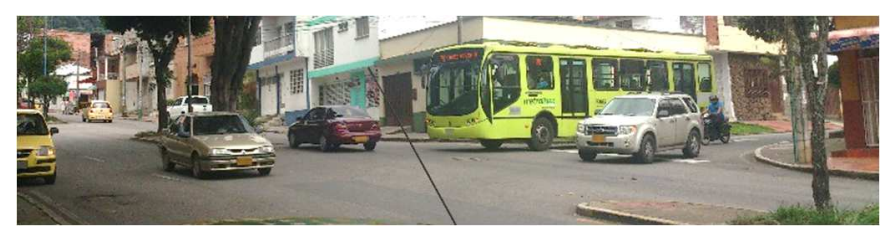

Figura 20. Intersección doble en la Calle 14 con Carrera 32A. Fuente: Autores.

\subsubsection{Infraestructura}

\section{Infraestructura vial:}

Comprende aspectos que van ligados a la movilidad del corredor; algunos de ellos son: tipo y calidad del pavimento, baches, señalización y amueblamiento urbano. En este documento únicamente se enfatiza en el tipo del pavimento y los baches presentes a lo largo del trayecto.

Durante un recorrido normal de la ruta en estudio se identifica la predominancia del pavimento flexible sujeto a ahuellamiento, grietas, y otras fallas de la estructura, 
Ingenierías USBMed, Volumen 7, No. 2, Julio-Diciembre 2016

producto de la baja capacidad de carga a comparación del pavimento rígido presente en el carril exclusivo, desde la estación Lagos hasta la estación Diamante y frente a las estaciones del SITM.

Por otro lado, a lo largo del corredor se encuentran diez baches, la mayoría de ellos presentes en el carril mixto de la Carrera 33, que, aunque no afecte directamente al vehículo del SITM, influye en la disminución de la velocidad de automóviles mientras es superado el obstáculo y, por consiguiente, provoca un aumento en el tiempo de viaje del bus que cubre la ruta P8. Un ejemplo de la presencia de baches en la trayectoria de los vehículos se evidencia en la Figura 21.

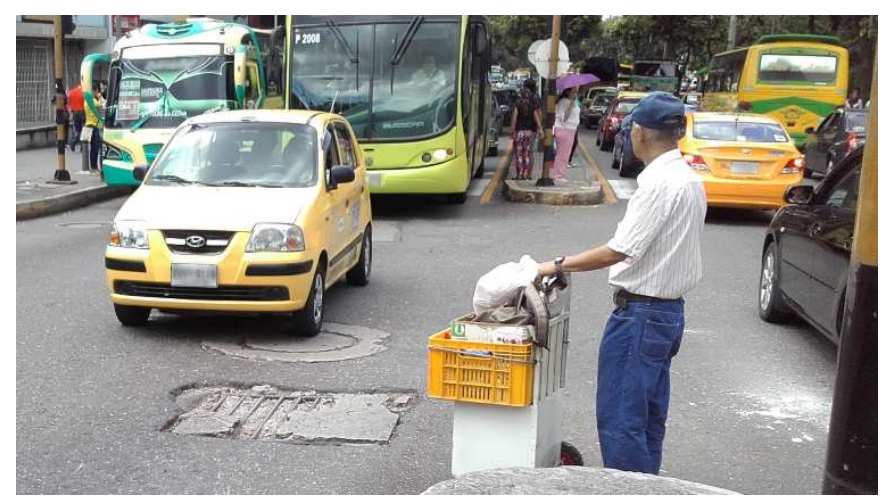

Figura 21. Presencia de un bache en la Carrera 33 con Calle 52. Fuente: Autores.

\section{Infraestructura del sistema:}

Como parte de la recopilación de datos, se observa que la infraestructura del sistema es esencial para el funcionamiento de un servicio expreso.

Por esta razón se identifica que la ruta P8 del SITM Metrolínea posee 99 puntos de parada a lo largo de su trayectoria, de las cuales el $29 \%$ se realizan en una estación (vagón) y el $71 \%$ restante en paradas demarcadas. La Tabla 3 muestra la distribución de los puntos de parada de la ruta P8 y generalidades de su servicio.

Para efectos de este documento se han denominado estaciones de transferencia a aquellas que son origen de rutas alimentadoras, ellas son: Estación Temprana, Lagos, Cañaveral, Provenza y Parque Estación UIS; y estaciones de puerta derecha a aquellas que su ingreso se realiza por las puertas laterales derechas del vehículo, validando su ingreso en la estación y no en el torniquete del bus (Figura 22).

Cabe aclarar, que el punto de parada origen de la ruta P8 (Portal del Valle), no cuenta con una estación de transferencia, ni con un vagón representativo del sistema; por el contrario, es un área en condiciones desfavorables destinada al estacionamiento de automotores.
Tabla 3. Distribución de puntos de parada de la ruta P8. Fuente: Autores

\begin{tabular}{|c|c|c|}
\hline $\begin{array}{l}\text { Puntos } \\
\text { parada }\end{array}$ & Tipo & $\begin{array}{l}\text { Horario } \\
\text { funcionamiento }\end{array}$ \\
\hline \multirow{3}{*}{$\begin{array}{l}\text { Estaciones. } \\
(29 \%)\end{array}$} & $\begin{array}{ll}\text { Estación } & \text { de } \\
\text { transferencia.(28 } & \% \\
\text { de estaciones) } & \\
\end{array}$ & Habitual al del SITM. \\
\hline & $\begin{array}{l}\text { Estación de puerta } \\
\text { derecha. ( } 24 \% \text { de } \\
\text { estaciones) }\end{array}$ & $\begin{array}{l}\text { Restringido en algunas } \\
\text { estaciones*. }^{*}\end{array}$ \\
\hline & $\begin{array}{l}\text { Estación. ( } 48 \% \text { de } \\
\text { estaciones) }\end{array}$ & Habitual al del SITM. \\
\hline $\begin{array}{l}\text { Paradas } \\
\text { demarcadas. } \\
(71 \%)\end{array}$ & Parada. (100\%) & Habitual al del SITM. \\
\hline
\end{tabular}

*Las estaciones de puerta derecha ubicadas en la Carrera 33, únicamente prestan el servicio de lunes a sábado entre las 10:00 a.m. y las 2:00 p.m. y entre las 4:30 p.m. y las 8:30 p.m., mientras que las demás prestan su servicio en el horario habitual del SITM.

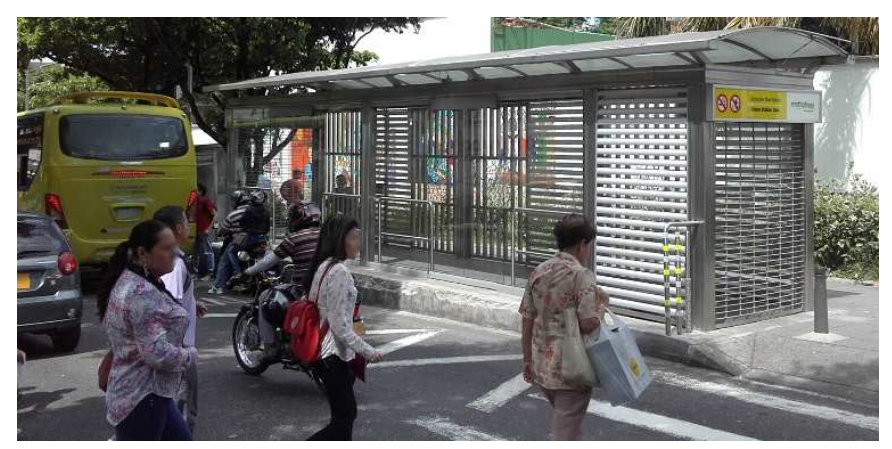

Figura 22. Estación Club Unión cerrada (Estación de puerta derecha) (Imagen del 05/07/2016 10:20 a.m.) Fuente: Autores.

\subsubsection{Ambiente de operación}

Esta sección está compuesta por algunos factores que intervienen en el nivel de servicio del corredor vial permitiendo un flujo vehicular regular durante un determinado periodo.

\section{Velocidad promedio entre puntos de parada:}

La velocidad promedio que se registró en el recorrido es de $30 \mathrm{~km} / \mathrm{h}$ aproximadamente; sin embargo, entre puntos de parada ubicadas en el carril mixto, la velocidad promedio encontrada fue alrededor de 25 $\mathrm{km} / \mathrm{h}$, y entre puntos de parada ubicados en el carril segregado de $40 \mathrm{~km} / \mathrm{h}$. Esto indica que efectivamente, el tramo exclusivo del SITM contribuye a que la velocidad del vehículo sea mayor en comparación a cuando transita por el trayecto denominado mixto.

Se observa que la velocidad de operación depende tanto del tipo como del número de carriles, ya que, por ejemplo: en horas valle, mientras en un tramo mixto de carril doble se estima una velocidad promedio de 40 $\mathrm{km} / \mathrm{h}$, en un tramo mixto de carril sencillo se registra un promedio de $24 \mathrm{~km} / \mathrm{h}$ en su velocidad.

Al mismo tiempo, en tramos mixtos de un día típico se determinan velocidades promedio de $27 \mathrm{~km} / \mathrm{h}$ en horas valle, mientras que el vehículo en el mismo tramo logra en promedio una velocidad de $18 \mathrm{~km} / \mathrm{h}$ en horas pico, lo 
que genera una necesidad de que, el vehículo que cubra la ruta en horas pico, encuentre un trayecto paralelo que permita disminuir su tiempo de viaje.

Es importante que la ruta pueda viajar lo más rápido posible debido a que velocidad es parte de la efectividad que se puede lograr en un servicio expreso.

\section{Estacionamiento de vehículos a lo largo del corredor:}

Adicionalmente, la velocidad promedio de recorrido está influenciada por el estacionamiento de vehículos en zonas donde está prohibido parquear.

En un recorrido realizado, en hora valle y día típico, se observaron 227 vehículos parqueados en el trayecto, en donde el $83 \%$ fueron particulares, incluidos los vehículos destinados para el transporte de mercancías urbanas estacionados en zonas comerciales, y el $17 \%$ restante conformado por vehículos de servicio público, todos ellos obstaculizando el paso de la ruta P8. Esta condición no es óptima, ya que, el ambiente de operación se ve congestionado producto de las maniobras que realiza el bus dentro del corredor tratando de superar estos vehículos parqueados. La Figura 23 evidencia esta situación.

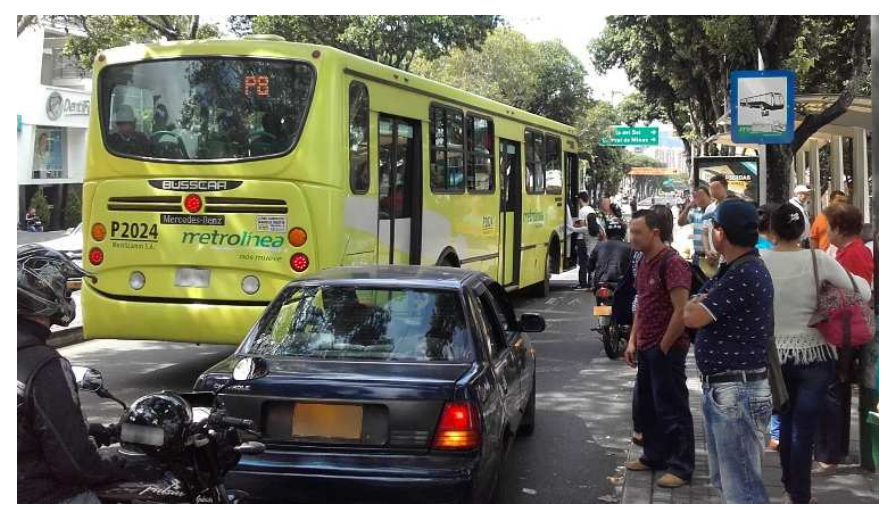

Figura 23. Vehículos obstaculizando el trayecto de la ruta P8. Fuente: Autores.

Además, en la Carrera 33 se evidencia que es la zona con mayor cantidad de parqueos en zonas prohibidas, y sumado a esto, los usuarios del transporte público colectivo convencional constantemente están ingresando o saliendo de estos vehículos, por lo que esa zona es bastante congestionada (Figura 24).

Como solución a la problemática generada por el transporte de carga, se debe buscar aplicar un modelo como el planteado por [18] que "permita integrar el transporte de mercancías con una buena distribución para establecer un orden y una armonía en la ciudad donde toda la sociedad se vea beneficiada", es decir, que la entrega de mercancías sea ordenada y eficiente que no interfiera con el transporte público y que aporte al desarrollo y crecimiento de la ciudad [18].

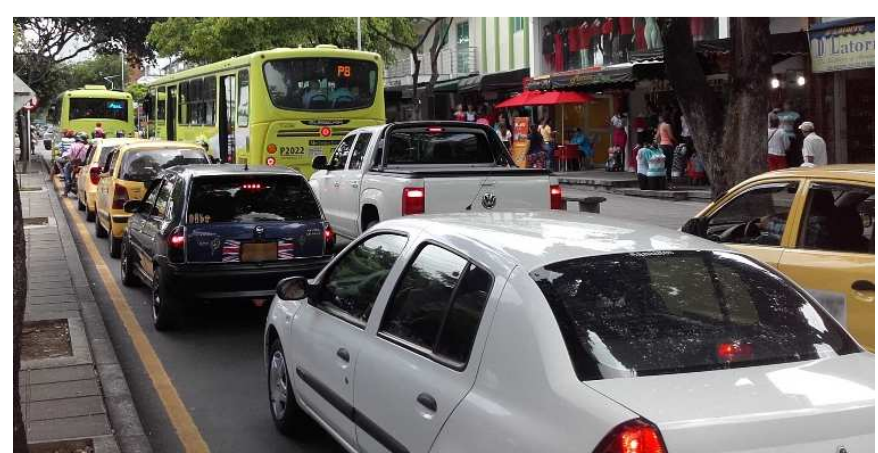

Figura 24. Congestión diaria en la Carrera 33. Fuente: Autores.

\section{Frecuencias de operación de la ruta P8 del SITM Metrolínea:}

La ruta P8 del SITM Metrolínea tiene despachos cada 10 minutos según se observa en Portal del Valle, punto de partida del servicio. Sin embargo, en el trabajo de campo realizado se observa que en el trayecto sur norte los vehículos mantienen este intervalo con más o menos dos minutos de retraso o adelanto, significando un $20 \%$ de error hasta la estación Diamante; de ahí en adelante se tienen errores mayores al $50 \%$.

Son varios los factores que influyen en que estas frecuencias no sean casi constantes a lo largo del recorrido; se considera que el hecho de que el tramo mixto sea en su mayoría de tan sólo dos carriles, sumado a la poca cultura ciudadana e inteligencia vial influye en que el tiempo entre vehículos que sirven la ruta P8 no sea casi uniforme.

\subsubsection{Tiempo de abordaje}

El tiempo de abordaje de los pasajeros es una de las principales causas para que el ciclo de recorrido de la ruta P8 aumente considerablemente; además, que la configuración del vehículo y la infraestructura del sistema contribuye a que el tiempo de abordaje sea elevado.

De la recopilación de datos se identifica que la diferencia entre el tiempo de abordaje en una parada y una estación está relacionada con la cantidad de usuarios que ingresan al vehículo y a su vez los que egresan. Es decir, en una parada, mientras 11 usuarios tardan aproximadamente 60 segundos en ingresar por el torniquete ubicado en la puerta delantera derecha del vehículo; en la estación, la misma cantidad de usuarios ingresan en 30 segundos por las puertas laterales izquierdas del bus, siempre y cuando no se presenten demasiados usuarios que egresen; en caso de haberlo, aquellos que ingresan deben esperar a que los usuarios egresen del vehículo, mientras que esta situación no se ve afectada en las paradas demarcadas porque la puerta de salida de los usuarios no es común con la de entrada.

Se identificaron puntos de parada como: Papi Quiero Piña y Cabecera del Llano con alta demanda de usuarios en las que el tiempo de abordaje es muy elevado y afecta directamente el tiempo del ciclo de recorrido (Figura 25). Lo que indica, que si el servicio expreso 
contempla estas paradas la necesidad de las estaciones sería de gran utilidad para disminuir los tiempos de viaje.

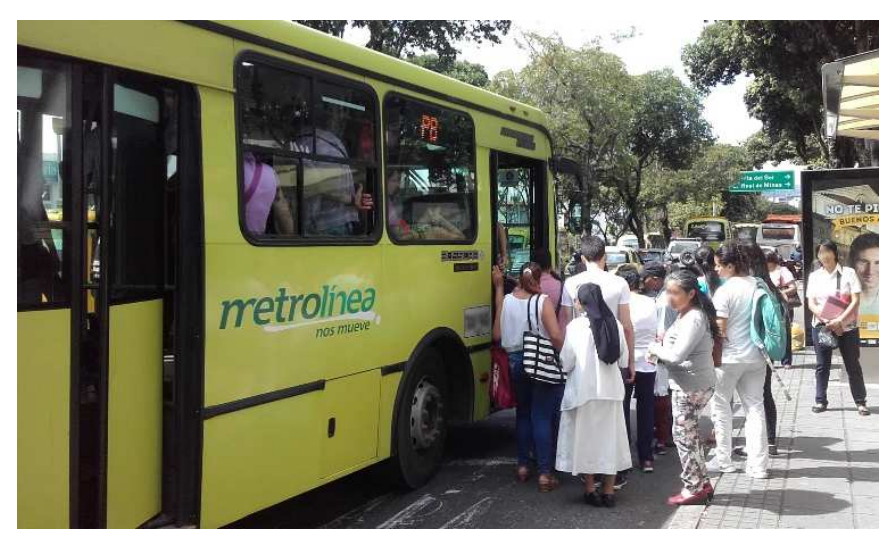

Figura 25. Congestión en el ingreso de usuarios al vehículo en una parada del trayecto. Fuente: Autores.

\subsubsection{Comportamiento de los usuarios}

El comportamiento de los usuarios es un factor que contribuye a la efectiva implementación de un servicio expreso, es por ello que mediante la encuesta realizada se analizaron algunos parámetros a tener en cuenta, ante una eventual implementación de un servicio expreso que se adapte a las necesidades y exigencias del usuario.

\section{Transbordos:}

En base a la información proveniente de las encuestas realizadas, el $71 \%$ de los encuestados estarían dispuestos a hacer un trasbordo con el fin de acceder a un servicio expreso, en su mayoría sustentando que su tiempo de viaje se reduciría considerablemente, comprobando así, que la confiabilidad del servicio expreso es fundamental para la efectiva implementación del mismo [11]. El $28 \%$ no está dispuesto a hacer trasbordo, afirmando que no lo harían porque se demorarían más tiempo, por pereza, incomodidad o porque la ruta al momento de tomarla estaría muy congestionada.

\section{Frecuencias:}

Se plantea un caso en el que el posible servicio expreso sea menos frecuente que la ruta P8 y se indaga a los encuestados si estarían dispuestos a esperar el nuevo servicio. Al revisar los resultados, se aprecia que el $64 \%$ de los usuarios no están dispuestos a esperar un servicio expreso debido a deducen que se demorarían más, o que tomarían el primer bus que pase, ya sea, el servicio expreso o la ruta de la cual se deriva.

Se estima que, si el SITM controla factores como tiempos de espera, frecuencias y capacidad de carga de usuarios, entre otros; los pasajeros serán más receptivos en realizar los transbordos necesarios para utilizar el servicio expreso.

\section{Conclusiones}

Se realizó el análisis preliminar para un servicio expreso derivado de la ruta P8 del SITM identificando: posibles modalidades de operación, condiciones actuales del SITM y del área metropolitana y comportamiento de los usuarios.

La muestra que se seleccionó al azar se distingue por tener un elevado número de personas menores a 30 años y a su vez, los viajes registrados están asociados, en su mayoría, con fines académicos. En base a lo anterior expuesto, se debe considerar a futuro la disminución del flujo de usuarios en los recesos académicos. Sin embargo, considerando la sección 4.2 de esta investigación, no se puede descartar la suspensión del servicio en los fines de semana.

También se encontró que el $71 \%$ de los encuestados realizan únicamente dos viajes diarios, lo que indica que el servicio expreso tendrá mayor eficiencia en los horarios habituales de entrada (mañana) y salida (tarde) de los diferentes centros de educación y lugares de trabajo, advirtiendo que en horas valle, el flujo de usuarios en el servicio expreso podrá disminuir significativamente y llegar a realizar viajes con un mínimo de pasajeros, situación no óptima para los servicios expreso.

Se comprobó que la confiabilidad del servicio influye en el comportamiento del usuario; dicho de otra manera, se verificó que uno de cada dos pasajeros no está dispuesto a esperar el servicio expreso si percibe que la operación del sistema es deficiente. Además, un poco más del $25 \%$ de los encuestados se niegan a realizar transbordo, y aquellos que están dispuestos, lo hacen confiando en que las frecuencias y los tiempos de espera y de viaje del servicio son mínimos. En vista de los resultados obtenidos se determinó que un servicio expreso sólo tiene influencia en las rutas que cubren el mismo corredor, mas no en aquellas que tengan trayectorias adyacentes.

Aplicar nuevos desarrollos en sistemas de transporte [9], en los que por medio de controladores de software y redes de comunicación se monitorean y controlan factores, como tiempos de despacho y parada, presentaría un panorama desfavorable, debido a que en su mayoría el recorrido de la ruta en estudio se realiza en un carril mixto donde el ambiente de operación es variable e impredecible.

En lo que se refiere a las modalidades propuestas para un servicio expreso se consideraron todos los casos posibles recopilados en la revisión de literatura y se identificó que todos ellos son aplicables a la ruta en estudio. Sin embargo, la eficiencia de cada modalidad propuesta está condicionada a factores indispensables únicos de cada esquema operacional, por ejemplo: la creación o adecuación de un retorno en cada extremo de los corredores de las rutas que sirven bajo la modalidad short turn; y la búsqueda de una vía menos congestionada que permita el pronto regreso de los vehículos que laboran en deadheading.

Se logró identificar los factores principales de la Tabla 1 que afectan los tiempos de viaje de tránsito en la operación de la ruta P8. Más allá de evaluar los factores 
que no pueden ser controlados por el operador, como el ambiente de operación, se evaluaron aquellos que sí pueden ser controlados como los despachos y la infraestructura del sistema, encontrando deficiencias y fallas. La Figura 25 evidencia la necesidad de la creación de estaciones en los puntos de parada que presentan un flujo diario de pasajeros alto, que permita disminuir el tiempo de abordaje. Al mismo tiempo de la creación de estaciones, el funcionamiento de cada una de ellas es indispensable para agilizar el ingreso y egreso de pasajeros; sumado a esto, los horarios de servicio restringidos que se presentan en las estaciones puede provocar el empeoramiento del problema en los tiempos de abordaje, y a su vez, aumentar la insatisfacción e inconformidad del usuario, como se evidenció en la Figura 10.

En último lugar, tanto los municipios que conforman el área metropolitana, como el esquema operacional del SITM Metrolínea, presentan constantes modificaciones. En el caso de los municipios, se evidencian cambios en los sentidos, ampliaciones viales y nuevos proyectos de infraestructura vial que buscan aliviar los problemas de movilidad. En cuanto al SITM, las variaciones se dan debido a que es un sistema joven (con 6 años de funcionamiento) que constantemente busca lograr una mayor efectividad en su servicio. Por ejemplo, durante esta investigación, se inició la modificación del tramo entre la estación Provenza y la parada Colegio la Merced, con el fin de añadir más carriles a este segmento del corredor (Bucaramanga) y la construcción del Intercambiador San Francisco (Piedecuesta); asimismo, el 1 de marzo de 2016, la ruta P7 salió de operación, producto de la implementación del primer servicio expreso en el área metropolitana de Bucaramanga.

\section{Trabajos futuros}

El análisis preliminar es el primer paso para la implementación de un servicio expreso, por lo que se recomienda profundizar individualmente en diferentes estudios que superen el alcance de esta investigación.

En primera estancia es importante que se revise por separado cada modalidad y que se ejecute un análisis detallado que permita identificar con mayor precisión las necesidades de cada una de ellas, para una eficiente implementación de una ruta expreso; un ejemplo, es realizar un estudio enfocado en la modalidad deadheading para determinar el sentido más cargado del corredor y el horario en que se presenta ese desbalance; de igual manera, realizar un estudio de movilidad para establecer una trayectoria alterna, paralela al corredor de la ruta en estudio, que presente mejores condiciones de tránsito, y así, evitar que el vehículo sea afectado por la congestión que presenta el corredor actual.

Más allá de estudiar las condiciones de operación de un servicio expreso, se debe conocer el impacto económico relacionado con la puesta en marcha del nuevo servicio. Para ilustrar esto, los costos que se generan en modificar una ruta existente, no son los mismos en la implementación de una nueva ruta, ya que esta última, requiere el aumento en el número de vehículos, la ampliación del recurso humano, y otros costos generados por la nueva operación.

Por su parte, se propone aplicar la investigación realizada por [18] con el fin de conocer el impacto que generaría una mejora del transporte de mercancías en la movilidad del corredor del servicio expreso.

Finalmente, se propone reestructurar y ejecutar una encuesta que permita un mejor seguimiento del flujo de los usuarios en el SITM, conocer factores conductuales de los usuarios, y a su vez, ampliar la muestra de estudio, ya que se infiere que con una mayor población se logra una mejor confiabilidad de respuesta.

\section{Agradecimientos}

Los autores reconocen los agradecimientos al director del grupo de investigación Geomática de la Universidad Industrial de Santander, Ing. Hernán Porras Díaz, PhD, por su acompañamiento; de igual manera, a los aportes generados por los coordinadores del diplomado DUNTI, en especial por la Ing. Claudia Patricia Báez y el Ing. Jesús Escalante, los cuales permitieron desarrollar este documento de manera organizada.

\section{Referencias}

[1] Departamento Nacional de Planeación, «Política Nacional de Transporte Urbano y Masivo (Documento CONPES 3260)». DNP, Bogotá D.C., Colombia, p. 36, 2003.

[2] H. Larrain Izquierdo, «Diseño de servicios expresos para buses», Pontificia Universidad Católica de Chile, 2013.

[3] A. M. El-Geneidy y J. Surprenant-Legault, «Limited-stop bus service: An evaluation of an implementation strategy", Public Transp., vol. 2, n.o 4, pp. 291-306, 2010.

[4] N. C. Silverman, «Limited-Stop Bus Service at New York City Transit», J. Transp. Eng., vol. 124, n.o December, pp. 503-509, 1998.

[5] S. Schwarcz, «Service Design for Heavy Demand Corridors: Limited-Stop Bus Service", Massachusetts Institute of Technology, 2004.

[6] H. Larrain Izquierdo, J. C. Muñoz A., y R. Giesen E., «Diseño de servicios expresos para el Corredor Av. Grecia en Santiago.» Santiago de Chile, p. 19, 2011.

[7] J. de Cea Ch., J. E. Fernández L., y I. Norambuena M., «Una metodología para el diseño topológico de sistemas de transporte público urbano de pasajeros", en XI Congreso Chileno de Ingeniería de Transporte, 2003, pp. 219-231.

[8] A. Ceder y N. H. M. Wilson, «Bus network design», Transp. Res. Part B, vol. 20, n.o 4, pp. 331-344, 1986.

[9] J. A. Kinirty, «Métodos formales para verificar los nuevos desarrollos en servicios de transporte», Ing. USBMed, vol. 2, n.o 1, pp. 15-17, 2011.

[10] D. A. Hensher, P. Stopher, y P. Bullock, «Service quality developing a service quality index in the provision of commercial bus contracts», Transp. Res. Part A Policy Pract., vol. 37, n.o 6, pp. 499-517, 2003.

[11] A. T. Murray y X. Wu, «Accesibility tradeoffs in public transit planning», J. Geogr. Syst., vol. 5, pp. 93-107, 2003.

[12] V. R. Vuchic, Urban Transit: Operations, Planning and Economics. Hoboken, N.J.: J. Wiley \& Sons, 2005.

[13] P. G. Furth y F. B. Day, «Transit Routing and Scheduling strategies for Heavy Demand Corridors", Transp. Res. Rec., vol. 1011, pp. 23-26, 1984. 
[14] C. Leiva, J. C. Muñoz, R. Giesen, y H. Larrain, «Design of limitedstop services for an urban bus corridor with capacity constraints", Transp. Res. Part B Methodol., vol. 44, n.o 10, pp. 1186-1201, 2010.

[15] H. Scorcia, «Design and Evaluation of BRT and Limited-Stop Services», Massachusetts Institute of Technology, 2010.

[16] H. Larrain, J. C. Muñoz, y R. Giesen, «Generation and design heuristics for zonal express services», Transp. Res. Part E Logist. Transp. Rev., vol. 79, pp. 201-212, 2015.
[17] Grupo de Investigación Geomática - Universidad Industrial de Santander., «Mapas del área metropolitana de Bucaramanga en software ArcGIS». Bucaramanga.

[18] M. Arroyave Rivera y V. Velasco Naranjo, «Desarrollo de un modelo de distribución urbana de mercancías con plataformas logísticas aplicado a la ciudad», Ing. USBMed, vol. 5, n.o 1, pp. 67-76, 2014 